\title{
A QUALITATIVE STUDY ON PSYCHOLOGICAL WELLBEING OF NURSES WORKING IN PALLIATIVE WARD
}

\author{
NELAVATHI A/P MARIMUTHU ${ }^{1}$, SITI SALINA ABDULLAH ${ }^{1,2^{*}}$ \\ ${ }^{1}$ School of Social and Economic Development, Universiti Malaysia Terengganu, 21030 Kuala Terengganu, \\ Terengganu, \\ ${ }^{2}$ Institute of Tropical Biodiversity and Sustainable Development, Universiti Malaysia Terengganu, 21030 Kuala, \\ Terengganu.
}

Corresponding author: ctsalina@umt.edu.my

\begin{abstract}
The main purpose of this research is to understand the experiences and psychological wellbeing of nurses working in palliative ward. There were two research questions driving this research throughout the process of data collection and data analysis. Four nurses from the palliative ward participated in this research to provide details on their psychological wellbeing. Semi- structured interview questions together with observation and simple survey questions were used to gather the data. Thematic analysis was used to identify the meaningful themes for each research question. Six main themes were identified: autonomy, environmental mastery, positive relation, personal growth, selfacceptance and purpose in life. The findings are highly relevant with the theory and literature. The findings indicate that the participants have good psychological wellbeing due to their huge experiences in palliative ward.
\end{abstract}

Keywords: Nurses, wellbeing, palliative ward, qualitative research

\section{Introduction}

In today's health care system, nurses are prone to a series of stress and depression while working with patients. When compared to other health service providers, nurses face greater stress, and unmanageable stress leads to burnouts, poor quality of care and high turnover among nurses in health care settings (Purohit and Vasava, (2017). According to Sharma et al. (2016), nurses play a significant direct role in delivering high quality service to patients, and nursing is as a complex profession that comprises both emotional and physical labour. Nurses also act as communicators, advocators, educators and caregivers to improve the health of patients in the best way possible. It is essential for nurses who are working in healthcare to have a stress-free attitude or good stress management skills because they are in an ideal position to enhance patient's resilience and empowerment, especially for nurses in palliative care (Ruddick, 2013). Palliative care was introduced to provide support and improve the quality of life of patients and their family. Palliative care intends to support patients with chronic health problems to live as actively as possible until their death (Sue, Hristina, \& Higginson, 2011). In dealing with the issues, palliative care faces several barriers such as lack of awareness, lack of professional training and effectiveness. This indicates that it is important to pay attention to nurses in the healthcare setting who are facing challenges while performing their duties. Focusing on nurses' psychological well-being while they are working in the healthcare system (palliative care) is important in order to understand this phenomenon accurately as their experience working in the healthcare setting may differ and thus their stressor and stress management skills cannot be the same. Therefore, this study intends to understand nurses' psychological wellbeing while they provide support and services to patients in a palliative ward.

\section{Materials and Methods Population and Sample}

In Malaysia, only several hospitals have palliative care and services including in-patient palliative care, consultative palliative care and community palliative care. This type of research targets nurses who act as caregivers to patients in a palliative ward in order to obtain information to achieve the research objectives. Hence, in this study, the targeted population was nurses working in palliative wards in hospitals throughout Malaysia, specifically palliative care nurses in Hospital Kuala Lumpur. The sample for this study was chosen from the palliative ward in Hospital Kuala Lumpur. Hospital Kuala Lumpur is one of the hospitals that has attached in-patient palliative ward together with its special palliative professionals. Hospital Kuala Lumpur also has its own facilities and a conducive environment for the patients. Even though other hospitals have palliative care, most do not have an in-patient ward. Therefore, the palliative ward in Hospital Kuala Lumpur provided a better chance of getting access to experienced nurses to explore their psychological well-being. At the time of the study, there was a large number of nurses working in Hospital Kuala Lumpur's palliative ward including senior nurses and new nurses. 


\section{Research Design}

In order to understand the experiences and psychological well-being of nurses working in the palliative ward, a qualitative research design was employed in this study. Hammersley (2013) described qualitative research as a way to study the real complex world in a more meaningful way, observing behaviors and encouraging interviewee expression rather than relying on closed-box questionnaire. The use of a qualitative method helped the researcher to explore palliative nurse's psychological well-being thoroughly and analyze common themes among their experiences. The case study design was deemed appropriate to explore a palliative nurse's psychological well-being and experiences in depth and in its natural context. In this research, semi structured interviews were used to obtain relevant information on the psychological well-being of palliative nurses. The semi-structured interview protocols were adapted from Ryff's multi-dimensional psychological well-being scale, with some amendments made to suit the research design and objectives. According to Creswell (2003), semi structured questions will lead the researcher to understand the complexity of the issues in the participant's view rather than narrowing meanings into several categories which may not give an in-depth understanding of the issue. Thus, the researcher chose a semi-structured design in constructing interview questions which focused on the six main domains of psychological well-being namely self-acceptance, purpose in life, positive relation with others, environmental mastery, autonomy and personal growth. There were three questions under each domain which were intended to explore the psychological well-being of palliative nurses and their experiences with patients, colleagues and the environment while working in the palliative ward of Hospital Kuala Lumpur. Besides that, an observation on the real setting also took place using systematic observation form in order to perceive the psychological well-being of nurses and their experiences in the palliative ward. A simple survey known as a patient's diary was given to participants to note their feelings every day in order to know more on the affective aspect. Before the interview session began with the palliative nurses, a demographical form was distributed and collected beforehand in order to obtain the personal information of the nurses that was required for this study such as their working period in the palliative ward. The informed consent forms (and recording consent forms) were given. All the data collected in this research were kept securely under the researcher's responsibility. The data (recordings) were completely erased after all the procedures of the research were completed, and the data would be transferred into intellectual products. Expected duration for data storage is one year from publications.

\section{Data Collection Procedures}

Upon obtaining approval from the National Medical Research Registry, Ministry of Health, Head of Palliative Department and the Hospital Director, the Participants Information Sheet (PIS), the informed consent form and a demographic form for the nurses were distributed to the participants to fill up prior to the interview. Participant recruitment process took place according to the requirement of this study. Each participant was interviewed twice and the interviews were conducted separately. The first participant was interviewed for the first session using semi-structured questions to understand a nurse's psychological well-being and experiences working in a palliative ward. After the first session, the interview transcript and coding process took place. During the interview sessions, observations in the real setting took place. After the researcher had obtained rich and saturated information on the nurses' experiences and psychological well-being, data collection was stopped, and data analyzed.

\section{Results}

The participants' ages ranged from 27 to 36 years. All participants were female and from Malay ethnicity. Half of them were married while others were single. The length of work experience ranged from 5 to 14 years. Table 1 shows the demographic of the participants.

Table 1: Demographic of Participants

\begin{tabular}{llllll}
\hline Participants & Age & Race & Gender & Marital Status & Years of Experience as a Nurse \\
\hline P1 & 27 & Malay & Female & Single & 5 \\
P2 & 30 & Malay & Female & Married & 8 \\
P3 & 31 & Malay & Female & Single & 8 \\
P4 & 36 & Malay & Female & Married & 14 \\
\hline
\end{tabular}




\section{Psychological Well-being of Palliative Nurses}

The data from the interview transcripts was analyzed and subsequently categorized into six main themes: Autonomy, Environmental Mastery, Positive Relation, Personal Growth, Self-Acceptance and Purpose in Life. Each theme had several subthemes. Direct quotes from the participants were highlighted to support and illustrate the theme.

\section{Theme 1: Autonomy}

Participants' responses to interview questions on autonomy suggested that palliative nurses have a higher level of autonomy while providing services to patients. This can be seen from the four subthemes that were identified from the transcripts. Three of the participants mentioned that they had a higher level of autonomy in all the four subthemes: voice out power, strong decision making, confidence level and teamwork.

Throughout the interview, the participants commonly shared that they did have chances to voice out their opinion including on controversial matters. They could share their knowledge in specific cases or with doctors and colleagues. The participants mentioned that sometimes their opinions were not really accepted by the doctors, but this did not affect their power to voice out. The participants mentioned that they would mostly react according to their preferences when giving opinions. This can be seen from the direct quotes of the participants. On having power to voice out, P1 remarked,

"Can voice out opinion any time" (L28).

"Sometimes they heard and sometimes not. If they heard, then okay" (L43)

"When I speak my juniors will listen" (L80)

The second subtheme mentioned by the participants while discussing their autonomy was their strong decision making skills. Three of the participants spoke about their strong decision-making skills while providing services to patients in the palliative ward, while one of the participants admitted not have strong decision-making skills, preferring to accept other people's opinions. The other participants mentioned that they always chose their own opinions in making decisions.

P1 remarked,

"If you want to follow me, just follow. But if you think I'm wrong then you don't have to follow."

(L113)

Confidence level was the most common subtheme mentioned by all the participants while explaining their autonomy. Usually, their confidence level was high while providing services to the patients, which helped them to give better services.

P4 said,

"I'm not grandiose but I think I'm a confident person and able to work with other people not only the doctors but all the health personnel and specialist." (L79-L81).

Teamwork was the forth subtheme commonly discussed when exploring the topic of autonomy. All participants talked about the importance of teamwork while providing good services to their patients.

On teamwork, $\mathrm{P} 2$ remarked,

"In critical situation sometimes we not, I don't make my own decision but if I have, in ward we have many staff nurse many nurses so during critical situation when just cooperate with them." (L69L71)

\section{Theme 2: Environmental Mastery}

The second theme, environmental mastery, was among the important themes that helped explore nurses' psychological well-being while working in the palliative ward. Throughout the interview, participants shared their environmental mastery in different aspects, and three subthemes were discovered that could provide a more systematic understanding which were Comfortableness, Controlling Difficulties, and Challenges. In general, the participants suggested that they had moderate environmental mastery while providing services to the patients in the palliative ward. Two participants claimed to have complete environmental mastery, while the other two limited environmental mastery.

While exploring participant's environmental mastery, comfortableness was one of the meaningful subthemes which provided a better understanding of their psychological well-being. P3 mentioned that her experiences had made her comfortable when she remarked, "I sit here almost more than 14 years and all ward I have been and go through. First class different second class also different. So, for the first time I'm in third class ward, seriously not comfortable." (L84-L87)

Controlling difficulties was another subtheme under environmental mastery which helped to answer the research questions. Two of the participants mentioned that they were able to control their difficulties and that these did not bring any detrimental effect to their service, while the other two participants (P1 and P2) mentioned emotional breakdown as a difficulty while providing services to terminally ill patients. P3 and P4 spoke about their ability to control difficulties in the palliative ward, which gave them satisfaction. Therefore, they did not find any difficulties in the palliative ward.

P4 shared her strategy when she remarked,

"Actually, life in a satisfying way, you need to balance everything like you cannot be too depressed, emotional, very happy and thinking about others life. You must balance your surrounding your professional.” (L116-L119) 
"I'm an optimist person and I can balanced everything in my life." (L128)

Challenges was the third subtheme often mentioned by participants while discussing their environmental mastery. All four participants faced their own challenges while arranging their lives. They shared different challenges and also how they managed them.

$\mathrm{P} 2$ remarked,

"It makes me very emotional especially when the patients are young." (L103-L104).

"I give time for me to set mind. Because sometimes we see a lot of patients so it's like, okay" (L111)

\section{Theme 3: Positive Relation}

Throughout the interview, the participants were asked to identify their positive relation while working in the palliative ward. From the data analysis, three subthemes were identified under positive relation, namely Relationship with Patients, Doctors and Colleagues; Willingness to Share; and Trust. Generally, all the participants had good relationships with the patients, but they had moderate trust and moderate willingness to share. This can be understood clearly through the data presentation of the subthemes.

Relationships with patients was shared by participants while discussing their positive relation in the palliative ward. All the participants had good relationships with the patients and doctors, even though sometimes they had disagreements with the doctors.

On her relationship, P1 remarked,

"Sometimes when they come for chemo, they will bring food. The patients live in kampung, they will bring rambutans and durians." (L270-L272)

Willingness to share was another subtheme commonly mentioned by the participants while discussing their positive relation. Two participants talked about their willingness to share their personal matters together with their colleagues while the other two participants did not prefer so due to some bad experiences such as being backstabbed. Participants who were willing to share their personal matters also had their differences in that.

P4 attested her willingness to share personal matters when she remarked,

"I'm a very straight forward person. So, if I want to share, I really share. I don't like to keep things because I don't want to burden. Normally, I will share whatever I have good." (L184-L186)

While exploring positive relations, trust was another meaningful subtheme shared by the participants. Two of the participants mentioned that they had trusting relationships while working in the palliative ward. $\mathrm{P} 4$ remarked,

"Yes, we have to trust in relationship and we cannot have that envy or doubt or personally. You have to see, not everything you can tell but certain things can ask for opinions." (L190-L192)

\section{Theme 4: Personal Growth}

Throughout the interview, personal growth was one of the meaningful themes explored in determining psychological well-being, from which emerged three subthemes, which were personalities, recognitions and development aspect. Generally, all the participants mentioned about their personal growth repeatedly throughout the interview sessions in different aspects. The detailed exploration can be seen from their direct quotes under each subtheme.

While exploring participants' personal growth, they commonly spoke about their personalities which had grown rapidly in a positive way. P1 remarked,

"Yes, I'm getting stronger. Last time I'm very shy to speak in English. I'm not very good in English but when I work here sometimes doctors speak English so what I do is I learned myself." (L338L340)

Recognition was another widely shared subtheme by all the participants while exploring their personal growth. They talked about it often when explaining the growth they had while providing care to the palliative patients. All the participants mentioned that their salary increased every year, which served as one form of recognitions.

P4 explained her recognition in personal growth when she remarked,

"Now, I'm a bachelor student" (L263)

"I'm an oncology nurse, specialized in that. Oncology and palliative." (L270)

While exploring personal growth, all the participants commonly mentioned about the development aspect, which become one of the important subthemes.

P1 remarked,

"I can really understand that they are not lying, they are in pain." (L359)

\section{Theme 5: Self-Acceptance}

Throughout the interview, another important theme of selfacceptance was explored in understanding the psychological well-being of nurses while providing services to terminally ill patients. There were three subthemes identified from the participants' responses which helped to understand their self-acceptance better and these were Accept-Themselves, Positive Feelings and Positive Personalities, and Satisfaction. All the participants showed a high level of self-acceptance and it can be seen clearly in the data presentation of the subthemes.

Accept-themselves was a commonly mentioned subtheme when exploring their self-acceptance. All the participants stated that they fully accepted their profession and it can be seen from their direct quotes.

P2 attested her self-acceptance when she remarked, 
"When I work here, it's like special care for the patients terminally ill because not all the nurse can do and know about chemo the medication in palliative care." (L166L168)

Positive feelings, the second subtheme, was shared commonly by all the participants when explaining their self-acceptance.

$\mathrm{P} 2$ remarked,

"First, I feel proud because not all the nurse can post in palliative ward. Special unit." (L235)

Besides that, positive personalities and satisfaction were the other meaningful subthemes that were widely shared by the participants while exploring their self-acceptance. P1 remarked,

"Sometimes, when we have problems at home, you need to put it outside and can put in expression. We need to hide that problem. If we mix that with job, we might do wrong and mad to patient." (L398L400)

\section{Theme 6: Purpose in Life}

Purpose in life was the last theme that explored the psychological well-being of nurses in order to answer the second research question. There were two subthemes under "Purpose in Life" which were Main Objective for Patients and Personal Goal. While exploring their purpose in life, participants commonly spoke about their main objective for the patients.

$\mathrm{P} 2$ remarked,

"My main objective being a palliative nurse is to give good care to the patient. Second is I want to upgrade my knowledge especially in palliative and oncology because I really love these department" (L259-L261)

While exploring their purpose in life, personal goal was one of the common and widely discussed subthemes by the participants. Three participants talked about their personal goals to achieve while in their period of service, while another participant did not have any personal goals. The personal goal shared by the three participants can be seen through their direct quotes.

P1 remarked,

year." (L431)

"I like and wanted to do post-basic. I think next

P2 remarked,

"First I want to get a post basic in palliative and oncology. Another one is I want to achieve in degree and masters, $\mathrm{PhD}$. $\mathrm{PhD}$ is my ambition, dream." (L283-L285)

"I want to teach my knowledge to all the nurse because I want here everyone give the good treatment. I also want everyone, my colleagues my nurse to have good knowledge because I don't want anybody look down to nurse." (L291-L294)

\section{Discussion}

Previous studies identified and highlighted that newly graduated nurses experience a high level of stress while trying to cope with the environment. The research findings also added on to that by showing similar experiences. The study participants shared that they experienced difficulties earlier on in their career and struggled to cope with that. According to Cheng (2015), nurses quit their job during their first job due to environmental stress caused by the profession. Besides that, Melnyk (2013) added on to that by saying, nurses, in early stages, lack resilience to cope with the occupational challenges which leads them to quit their jobs. A higher rate of quitting their jobs are found among newly graduated nurses compared to experienced nurses (McVicar, 2016). However, having five to fourteen years of service enables the participants to maintain their psychological well-being at a good level which does not disrupt their service in the palliative ward. According to Blomberg et al., (2014), a series of stress in the nursing profession begins since nurses graduated and join their first job. These conditions may develop when they are placed in critical wards such as the Intensive Care Unit (ICU) and Palliative Care. This is because, the stress level may differ according to the environment and workplace. The condition and patients in a palliative ward are severe than in other wards in terms of disease and health complication. For the participants, they managed the challenges and built positive relationships during their work. It helps boost their self-acceptance and purpose in life.

The theory of Maslow suggested five levels which will be achieved one by one before psychological wellbeing. These basic needs begin from physiological needs and are followed by safety, belongingness and esteem before self-actualization. All these needs can be related to palliative nurses based on themes discovered while trying to understand their psychological well-being. The findings show that nurses do not face any problem in achieving physiological needs as they receive salary and are grouped as ordinary peoples in society. Even though they withhold their opinions sometimes, it does not give any personal effect which causes their safety to be insecure. Therefore, they can continue their journey to the next level in the Maslow self-actualization hierarchy, which is belonging. The participants commonly shared that they love their patients and receive love from their patients as well. This shows that their developments are in parallel with the theoretical standard. Achieving belongingness leads them to acquire the following need which is esteem. This is highly related with the finding where the participant shared this as one of the meaningful subthemes; recognition under theme; personal growth. All the participants managed to receive the recognition in various aspect based on their qualification. These provide a wider chance for them to move to the following level which is self-actualization. 


\section{Conclusion}

Despite the challenges experienced during their service, palliative nurses seem to be at a healthy level of psychological well-being in general as has been suggested by the findings of this study. The six meaningful themes and several subthemes explain their level of well-being, together with their experiences as evidence. Their psychological well-being has improved steadily as they gain more experiences over time. Almost all the participants have gone through a difficult phase in maintaining their psychological well-being earlier on in their career. As nurses are considered being at high risk of work-related stress and reduced well-being, it is crucial for them to maintain their psychological well-being at a good level so as not to disrupt their service in the palliative ward.

\section{References}

Blomberg, K., Isaksson, A., Allvin, R., Bisholt, B., Ewertsson, M., Engstrom, A. K., Gustafsson, M. (2014). Work stress among newly graduated nurses in relation to workplace and clinical group supervision. Journal of Nursing Management, 24(1), 80-87.

Cheng CY, Liou SR, T. H. \& C. C. (2015). Job stress and job satisfaction among new graduate nurses during the first year of employment in Taiwan. International Journal Nursing Practice., 21(4).

Cresswell J.W. (2003). Research design: Qualitative, quantitative, and mixed methods approaches (2nd ed.). Thousand Oaks, CA: Sage.

Hammersley, M. (2013). What is Qualitative Research? What Is? Research Methods. London: Continuum/Bloomsbury.

McVicar, A. (2015). Scoping the common antecedents of job stress and job satisfaction for nurses (2000-2013) using the job demands- resources model of stress. Retrieved January 2, 2018, from https://onlinelibrary.wiley.com/doi/full/10.1111/jonm. 12326

Melnyk BM, Hrabe DP, S. L. (2013). Relationships among work stress, job satisfaction, mental health, and healthy lifestyle behaviors in new graduate nurses attending the nurse athlete program: a call to action for nursing leaders. Nursing Adm Q., 37(4), 278-285.

Purohit, B., \& Vasava, P. (2017). Role Stress among Auxiliary Nurses Midwives In Gujarat, India. Retrieved $\begin{array}{lll}\text { January } & 2, & \text { 2018, from }\end{array}$ https://bmchealthservres.biomedcentral.com/articles/1 0.1186/s12913-017-2033-6
Ruddick, F. (2013). Promoting mental health and wellbeing. Nursing Standard, 27(24), 35-39.

Sharma, J., Dhar, R. L., \& Tyagi, A. (2016). Stress as a mediator between work-family conflict and psychological health among the nursing staff: Moderating role of emotional intelligence. Applied Nursing Research, 30, 268-275.

Sue Hall, Hristina Petkova, A. D. T., \& Higginson, M. C. and I. J. (2011). Palliative care for older people: better practices. Denmark. Retrieved from http://www.euro.who.int/_data/assets/pdf_file/0017/1 43153/e95052.pdf 